\title{
Condensing osteitis of the clavicle: magnetic resonance imaging as an adjunct method for differential diagnosis
}

\author{
Marianne A C Vierboom, Julia D J Steinberg, Eduard L Mooyaart, Martin H van Rijswijk
}

Department of

Rheumatology,

Groningen,

The Netherlands

M A C Vierboom

M H van Rijswijk

Department of

Radiology,

University Hospital,

Groningen,

The Netherlands

J D J Steinberg

E L Mooyaart

Correspondence to: Dr Marianne A C Vierboom, University Hospital

Groningen,

Department of

Rheumatology,

Oostersingel 59

PO Box 30.001,

The Netherlands.
Then

Accepted for publication

30 July 1991

\begin{abstract}
Condensing osteitis of the clavicle is a benign disorder leading to osteosclerosis of the medial end of the clavicle. The differential diagnosis between condensing osteitis of the clavicle and ischaemic necrosis of the medial clavicular epiphysis (Friedrich's disease), osteoid osteoma, and low grade osteomyelitis can be difficult. In the case history reported here, magnetic resonance imaging was a useful non-invasive procedure for the diagnosis of condensing osteitis of the clavicle.
\end{abstract}

Condensing osteitis of the clavicle is a rare and benign disorder, manifested by a painful swelling at the medial end of the clavicle. It was first described by Brower et al in $1974 .^{1}$ Clinically and radiologically the differential diagnosis between condensing osteitis of the clavicle and ischaemic necrosis of the medial clavicular epiphysis (Friedrich's disease), osteoid osteoma, and low grade osteomyelitis can be difficult. As magnetic resonance imaging is reported to be an excellent diagnostic modality for the imaging of bone abnormalities, we studied a patient with possible condensing osteitis of the clavicle using magnetic resonance imaging.

\section{Case history}

A 37 year old woman with three young children was referred to the department of rheumatology with a 10 month history of a painful swelling of the medial end of the left clavicle. Raising the left arm above shoulder level aggravated the pain. There was no history of infection or trauma. She had no systemic complaints.

Examination showed slight tenderness and swelling over the medial end of the left clavicle. Abduction intensified the pain. No other abnormalities were found. The results of routine biochemical tests were within normal limits.

Plain radiography and polytomography (fig l) of the left sternoclavicular joint showed bony sclerosis of the caudal half of the medial end of the left clavicle without joint narrowing or destruction of the articular surface. A technetium-99m total body bone scan (fig 2) showed a unifocal, intense uptake at the medial end of the left clavicle.

The clinical and radiographic features suggested the diagnosis of condensing osteitis of the left clavicle. Non-steroidal anti-inflammatory drugs (NSAIDs) and limitation of physical activities were advised. During the following year the symptoms did not subside and the radiographic features remained constant. To exclude other causes of sclerotic bony lesions, such as ischaemic necrosis of the medial clavicular epiphysis (Friedrich's disease), osteoid osteoma, and low grade osteomyelitis, we advised an incisional biopsy of the clavicle. This

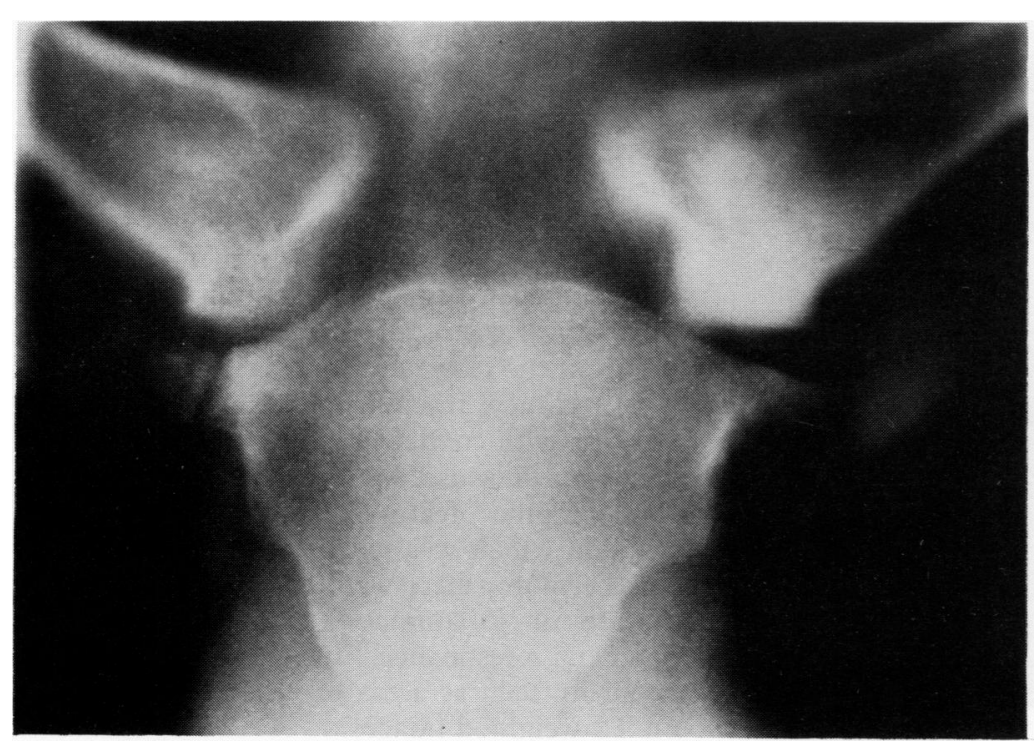

Figure 1 Tomography of the sternoclavicular region showing amorphous sclerosis of the caudal part of the medial end of the left clavicle. There is no narrowing of the joint or destruction of the articular surface.

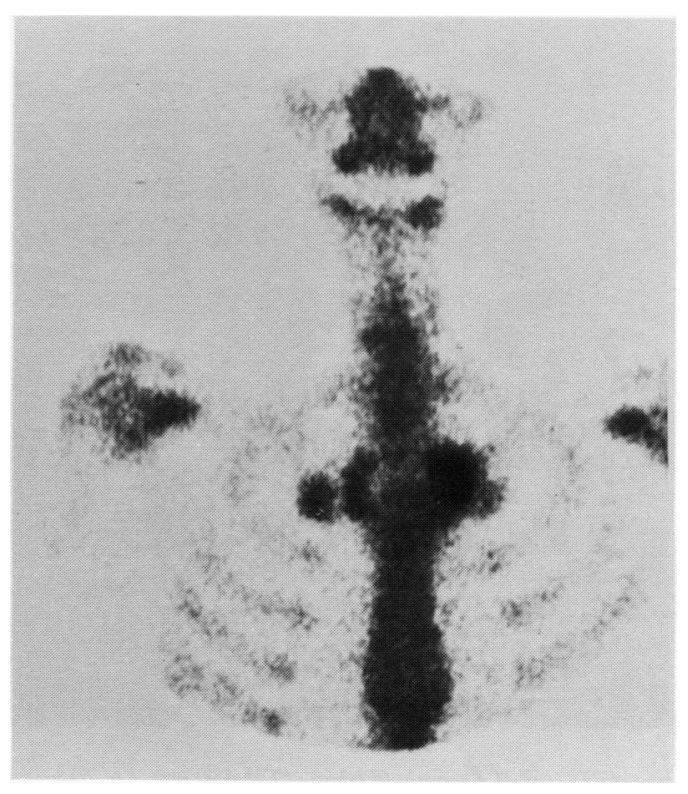

Figure 2 Technetium- $99 m$ bone scan. There is a region of high uptake at the medial end of the left clavicle. 
was refused by the patient. Therefore we performed a magnetic resonance imaging examination. T1 (TR $650 \mathrm{~ms}$, TE $20 \mathrm{~ms}$ ) and T2 (TR $2000 \mathrm{~ms}$, TE $100 \mathrm{~ms}$ ) weighted spin echo images of the sternoclavicular region were performed in the coronal plane on a $1.5 \mathrm{~T}$ magnet system using a surface coil and a 180 $\mathrm{mm}$ field of view. The T1 weighted images (fig 3A) showed an area of low signal intensity and an area with a complete lack of signal at the medial end of the left clavicle. There is a slightly thickened cortex. The T2 weighted images (fig 3B) showed low signal intensities in this region. Within these areas no nidus or double lines were seen. The soft tissue and joints were not affected.

The diagnosis of condensing osteitis of the clavicle could thus be confirmed. After continuation of treatment with NSAIDs and limitation of physical activities, the symptoms subsided over the next follow up period of six months.
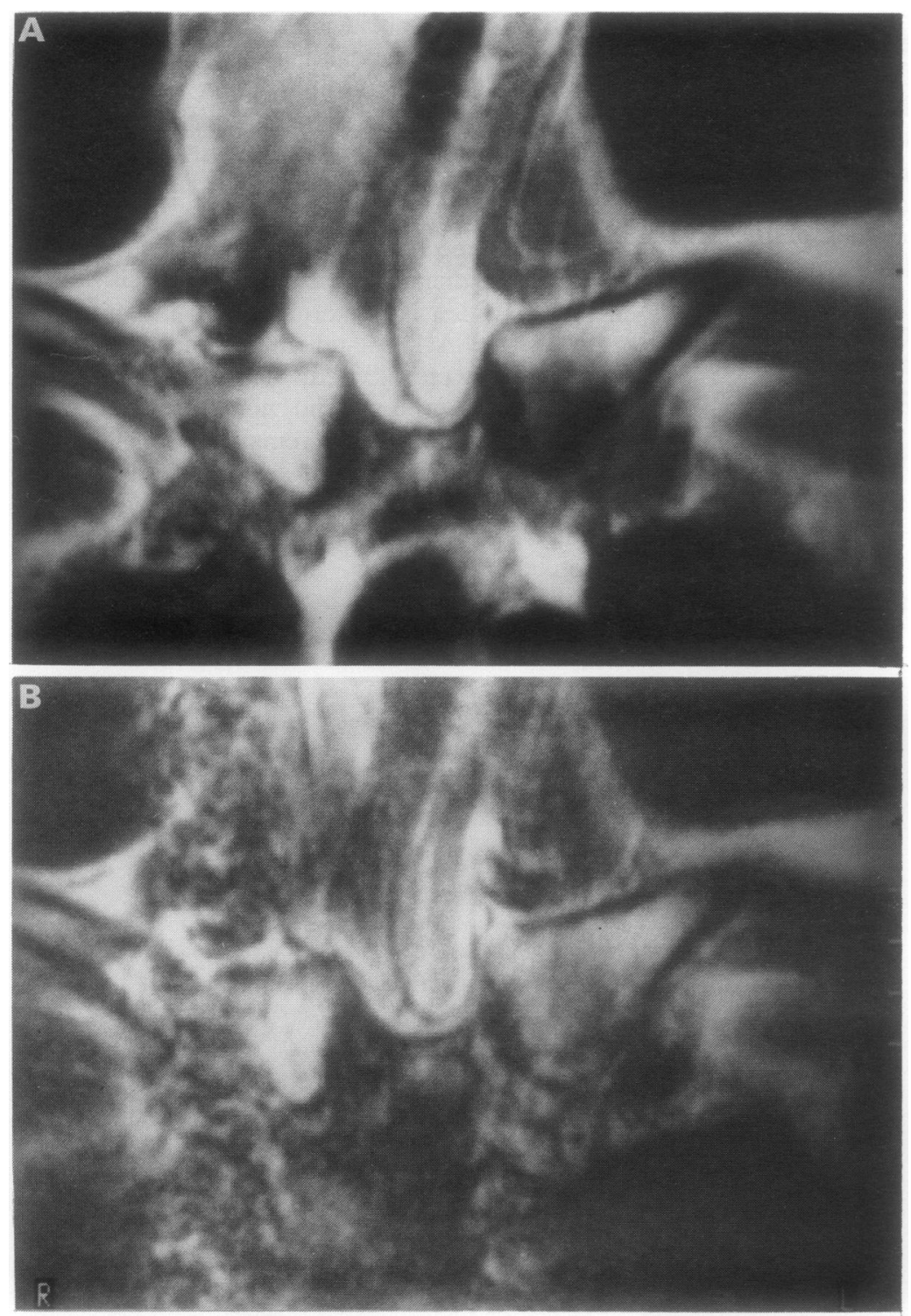

Figure 3 (A) Tl weighted image (TR $650 \mathrm{~ms}, T E 20 \mathrm{~ms}$ ) of the sternoclavicular region showing an area of low signal intensity and an area with no signal. There is a slightly thickened cortex. (B) T2 weighted images (TR $2000 \mathrm{~ms}$, TE $100 \mathrm{~ms}$ ) showing low signa intensities at the medial end of the left clavicle.

\section{Discussion}

Since the first description by Brower et al in $1974,{ }^{1}$ approximately 25 cases of condensing osteitis of the clavicle have been reported. Most of the patients are women of late childbearing age who have a painful swelling over the medial part of one clavicle. The pain is often referred to the ipsilateral shoulder and is aggravated by abduction of the arm. Usually there is a history of repeated mechanical stress. ${ }^{1-3}$ Systemic complaints are absent. Routine laboratory tests are within normal limits. Plain radiographs and tomograms reveal bony sclerosis and mild enlargement of the inferomedial aspect of the clavicle. In all cases described, the sternoclavicular joint and the adjacent soft tissues appear to be normal; however, technetium-99m bone scintigraphy shows an increased accumulation of the isotope in this region.

As the radiological features of condensing osteitis of the clavicle are usually distinctive, several workers recommend that an excisional or preferably an incisional biopsy should only be performed in patients with a doubtful clinical or radiological presentation. ${ }^{45}$ As the symptoms of our patient did not subside, we questioned our diagnosis of condensing osteitis of the clavicle, but the patient refused the proposed biopsy. As an alternative, we performed a magnetic resonance imaging examination. In our opinion, the most difficult entities to be excluded in this patient were ischaemic necrosis of the medial clavicular epiphysis (Friedrich's disease), osteoid osteoma, and low grade osteomyelitis.

There is a clinical similarity in Friedrich's disease and the radiographic features can mimic those of condensing osteitis of the clavicle, particularly in the first phase of the disease. ${ }^{6}$ However, we expect the magnetic resonance imaging findings of Friedrich's disease to be similar to aseptic bony necrosis in the other parts of the body-that is, a high signal intensity of the inner border parallel with a low signal intensity of the periphery, also known as the double line sign. ${ }^{78}$ In our patient no double line sign was seen.

Osteoid osteoma is a benign tumour of the bone that consists of a nidus of osteoid tissue with a highly vascular stroma, surrounded by reactive sclerosis. Radiological demonstration of the nidus may be difficult, even with tomography. Magnetic resonance imaging is extremely sensitive in showing the central nidus of high signal intensity in the surrounding low signal intensity of sclerotic bone. ${ }^{79} \mathrm{~A}$ nidus was not found in the clavicle of our patient.

The clinical resemblance of low grade osteomyelitis with condensing osteitis of the clavicle has been discussed by several workers. ${ }^{124}$ Radiographic features include a periostal reaction which was not present in our patient. Furthermore, there were no signs of soft tissue disease or inflammation in the marrow on magnetic resonance imaging examination, normally seen as areas of high signal intensity on $\mathrm{T} 2$ weighted images. ${ }^{7} 10$

Our diagnosis of condensing osteitis of the clavicle could therefore be confirmed. We conclude that if there is doubt about the clinical 
presentation and radiographic features of condensing osteitis of the clavicle, magnetic resonance imaging examination of the clavicle can be a discriminating non-invasive diagnostic procedure. This is the first time diagnosis of condensing osteitis of the clavicle by magnetic resonance imaging has been described.

1 Brower A C, Sweet D E, Keats T E. Condensing osteitis of the clavicle: a new entity. Am $\mathcal{F}$ Roentgenol 1974; 121: $17-21$.

2 Duro J C, Estrada P, Ribas D, Bartons S, Rotés-Querol. Condensing osteitis of the clavicle [letter]. Arthritis Rheum 1981; 24: 1454-5.

3 Cone R O, Resnick D, Goergen T G, Robinson C, Vint V,
Haghighi P. Condensing osteitis of the clavicle. Am Roentgenol 1983; 141: 387-8.

4 Kruger G D, Rock M G, Munro T G. Condensing osteitis of the clavicle: a review of the literature and report of three cases. F Bone foint Surg [Am] 1987; 69: 550-7.

5 Abdelwahab I F, Hermann G, Ramos R, Klein M J, Kenan $S$, Lewis M M. Case report 623. Osteitis condensans of the left clavicle. Skeletal Radiol 1990; 19: 387-9.

6 Linng G, Heinemeier G. Morbus Friedrich-Aseptische Knochennekrose des sternalen Klaviculaendes. Fortschr Röntgenstr 1981; 134: 74-5.

7 Holland B R, Freyschmidt J. Kernspintomographische Ergebnisse bei Knochenveränderugen mit Sklerose. Fortsch Röntgenstr 1988; 149: 513-21.

8 Ensign M F. Magnetic resonance imaging of hip disorders. Semin Ultrasound CT MR 1990; 11: 288-306.

9 Glass R B J, Poznanski A K, Fisher M R, Shkolnik A, Dias L. MR imaging of osteoid osteoma. F Comput Assist Tomogr 1986; 10: 1065-7.

10 Cohen M D, Cory D A, Kleiman M, Smith J A, Broderick N J. Magnetic resonance differentiation of acute and chronic osteomyelitis in children. Clin Radiol 1990; 41: 53-6. 